\title{
Administering of Local Anesthesia with Bupivacaine in the Patients Undergoing Inguinal Hernia Repair
} \author{
$\operatorname{zan}^{5 *}$ \\ ${ }^{1}$ Department of General Surgery, Faculty of medicine, Isfahan University of Medical Sciences, Isfahan, Iran \\ ${ }^{2}$ Department of Surgery, Faculty of medicine, Kashan University of Medical Sciences, Iran \\ ${ }^{3}$ Department of Neurology, Faculty of medicine, Kashan University of Medical Sciences, Iran \\ ${ }^{4}$ Department of Anesthesiology, Faculty of medicine, Kashan University of Medical Sciences, Iran \\ ${ }^{5}$ Department of Surgery, Faculty of medicine, Isfahan University of Medical Sciences, Iran
}

Mehrdad Hosseinpour ${ }^{1}$, Leila Gafoor ${ }^{2}$, Ebrahim Kouchaki ${ }^{3}$, Mohammadreza Razavizadeh ${ }^{4}$, Mohsen Kolahdou-

*Corresponding author: Mohsen K, Associate Professor, Department of surgery, Isfahan University of medical sciences, Isfahan, Iran, Tel: +98 31 36202020; +989131153035/ Fax: +98 31 36684510; E-mail: kolahdouzan@med.mui.ac.ir

\begin{abstract}
:
Background: postoperative pain is a most prevalence complication in herniorrhaphy whatever the management of this pain is a basic problem. The aim of this study was to compare the effects of pre and post incisional infiltration of the surgical area with bupivacaine on cortisol and prolactin release and postoperative pain in patients undergoing inguinal hernia repair.

Methods: In this clinical trial study, 48 adult male patients have undergone inguinal hernia repair and spinal anesthesia, as signed to 2 groups. Local anesthesia was performed by $2 \mathrm{mg} / \mathrm{kg}$ bupivacaine $0.5 \%$ while placebo group did not receive any local anesthesia. Plasma cortisol and prolactin were compared in both groups.

Results: In postoperatively, the plasma concentration of cortisol and prolactin in local anesthesia group were significantly lower than placebo group $(\mathrm{P}=0.001$ and $\mathrm{P}=0.013$, respectively). Visual Analog Scale (VAS) in local anesthesia group was significantly lower than placebo group in $12,24,48$ and $72 \mathrm{hrs}$ after operation $(\mathrm{P}<0.0001$, for all four $)$. Also, there was a significant correlation between the two groups according to rescues analgesic $(\mathrm{P}<0.0001)$.

Conclusions: The finding of the current study suggests that usage of local anesthesia is a safe, effective and feasible procedure as decreases the cortisol and prolactin hormones, postoperative pain and analgesic drugs consumption.
\end{abstract}

Keywords: Inguinal hernia; Cortisol; Prolactin; Postoperative pain

\section{Introduction}

Herniorrhaphy or inguinal hernia repairing is a painfulness and exigent operation which half of the patients have moderate to severe pain after surgery ${ }^{[1]}$. Postoperative pain is one of the most common surgical problem, this pain is created patient distress, prolonged treatment process, delay in returning to a normal level of activity and increasing the rate of hospitalization in after operation. Postoperative pain is associated with more complications such as nausea and vomiting. Its noteworthy, $80 \%$ of subjects have needed to rescue analgesic after hernia repairing ${ }^{[2]}$. Usually opioids are used to controlling of postoperative pain, however high dose of this drugs are associated with more adverse effects such as respiratory suppression, ileus, nausea and vomiting. On the other hand, reducing of opioids dosage may increase postoperative pain. Non-steroidal anti-inflammatory drugs (NSAIDs) are similar to opioid drugs regarding to pain controlling. NSAIDs have many side effects such as decreased hemostasis, renal dysfunction and gastrointestinal hemorrhage. Also, there are several ways to control postoperative pain which have been suggested to inguinal hernia repair such as ilioinguinal and iliohypogastric nerves blockage $^{[3]}$ and intraperitoneal bupivacaine injection ${ }^{[4]}$. Inguinal hernia repair under general and local anesthesia is a simple and effective method for postoperative pain control, this method is caused blocking of peripheral nerves and management of postoperative pain, its noteworthy that this operation method no have major or severe adverse effects ${ }^{[5]}$. The hypothalamic-pituitary-adrenal actions during injury are well known, especially these actions effect on the endocrine system and the release of substances such as catecholamines, glucocorticoids, growth hormone and glucagon, and suppression of insulin release. These hormonal mechanisms can change the catabolism and metabolism, evaluations showed
Received date: February 27, 2018

Accepted date: May 31, 2018

Published date: June 8, 2018

Citation: Kolahdouzan, M., et al. Administering of Local Anesthesia with Bupivacaine in the Patients Undergoing Inguinal Hernia Repair. (2018) J Anesth Surg 5(1): 71- 74 .

Copy Rights: (C) 2018 Kolahdouzan, M. This is an Open access article distributed under the terms of Creative Commons Attribution 4.0 International License. 
Citation: Kolahdouzan, M., et al. Administering of Local Anesthesia with Bupivacaine in the Patients Undergoing Inguinal Hernia Repair. (2018) J Anesth Surg 5(1): $71-74$.

that the body response during injury is associated with mortality and mobility in adult. Surgical trauma and postoperative pain change the stress hormonal secretion such as Adrenocorticotrophic hormones (ACTH), cortisol and prolactin ${ }^{[6-8]}$. Plasma concentrations of cortisol and prolactin remain high in several days after surgery and gradually reach to normal levels ${ }^{[9,10]}$. Also we have hypothesis that local anesthesia can decreases stress hormones in hernia repairing so, in this study, we evaluated the effects of local anesthesia and spinal anesthesia on the plasma concentration of cortisol and prolactin, postoperative pain and needed rescue analgesic in the patients undergoing herniorrhaphy.

\section{Methods and Materials}

\section{Patients}

In this randomized clinical trial double blinded, that was registered in Iranian registry of Clinical Trial (No: irct201107131106n4), 140 patients with inguinal hernia were reformed to Al-Zahra hospital, Isfahan, Iran and 48 men with the clinical diagnosis of a groin hernia have needed elective surgery were enrolled to study, patient's enrollment was between 2009 to 2014 (Figure 1). Also this study was approved in the Kashan University of medical Sciences-Isfahan-Iran. Inclusions criteria were included patients with inguinal hernia that have indication for elective surgery and have informed consent to participation in study, in addition Patients with hernias stuck (strangulation or incarceration) or requiring to emergency surgery, having a history of chronic diseases or the opioids using, not meet inclusion criteria. Also patients that treated with corticosteroids or steroid medications in during of therapy, had blood pressure (BP) lower than $100 \mathrm{mmHg}$ or higher than $140 \mathrm{mmHg}$ or bleeding with Pulse rate (PR) higher than 80 beats per minute in during of operation, not been followed up study and had side effect in the during of investigation were excluded. In this investigation, patients were randomly divided into two groups in before operation, randomization was according to OxMAR software, also this method was concealment, the sample size was 24 patients in each group. First group or local anesthesia group was under spinal anesthesia and local anesthesia, while Placebo group was under spinal anesthesia. In before operation, 5cc blood clots were taken of patients according to standard protocol and plasma levels of cortisol and prolactin were measured in both groups. All demographics information of subjects such as age and body mass index (BMI) was collected in check list.

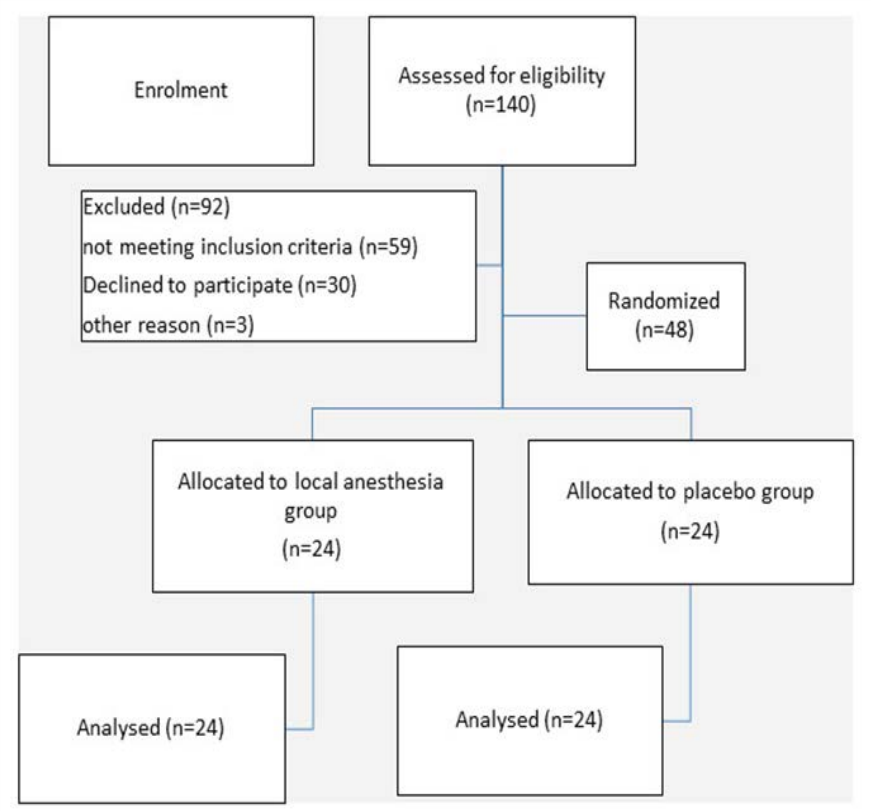

Figure 1: Consort diagram of patients through every stage of the study.

\section{Anesthesia}

Spinal anesthesia was performed for all patients according to standard protocol spine. Then spinal needle (Size No.25) was used to inject $3.5 \mathrm{cc}$ bupivacaine $0.5 \%$ in cerebrospinal fluid (CSF) and was injected in L4-L5. All operation were done by Lichtenstein technique ${ }^{[11]}$. In order to apply a local anesthetic, bupivacaine (half a percent to the rate of $2 \mathrm{mg} / \mathrm{kg}$ ) was injected in the subcutaneous between before the end of operation and after closing fascia.

\section{Assessment}

In after operation blood sampling was taken of patients and plasma level of cortisol and prolactin measured according to standard protocol. The postoperative pain was measured according to Visual Analogue Scale (VAS) (scored between 1 to 10) at onset, 12, 24, 48 and 72hrs after operation. Also needed rescue analgesic (Pethidine) was evaluated up to $72 \mathrm{hrs}$, after operation.

\section{Statistics}

Sample size was according to sample size estimation formula. In this regard, the confidence level and power detection were $95 \%$ and $80 \%$, standard deviation of VAS of local anesthesia in post operation was 1.3 and mean difference was $0.4^{[5]}$, so sample size was calculated 24 in each group. All data was entered to SPSS (version 24) software, the mean change of hormones in two groups was used of Independent T-test and Paired $\mathrm{T}$ test showed changing of hormones in pre and post operationally, Mann-Whitney test was used to relation of VAS scores in both groups. Also we used of Chi Squire Test. Number (percent) and mean \pm SD showed for all data, also p-value less than 0.05 was considered as significance threshold.

\section{Result}

In this study, the mean age of patients in the local anesthesia and placebo groups were $35.92 \pm 9.65$ and $35.46 \pm 9.61$ years, respectively, also the mean of BMI in the local anesthesia and placebo groups were $23.75 \pm 3.82$ and $24.69 \pm 3.17 \mathrm{Kg} /$ $\mathrm{m}^{2}$, respectively. Therefore, there was no significant difference between two groups regarding to age and BMI ( $\mathrm{P}=0.87$ and 0.40 , respectively) (Table 1 ). In preoperational, the mean plasma levels of cortisol (ug / dl) in local anesthesia and placebo groups were $20.24 \pm 5.18$ and $19.94 \pm 6.52$, respectively and the mean plasma levels of prolactin (ug / dl) in local anesthesia and placebo groups were $12.47 \pm 3.36$ and $11.84 \pm 2.76$, respectively. Therefore, there was no significant difference between both groups according to plasma level of cortisol and prolactin in preoperational ( $\mathrm{P}$-value were 0.17 for cortisol and 0.65 for prolactin). In post operational, the mean of plasma levels of cortisol in the local anesthesia and placebo groups were $13.96 \pm 3.64$ and $26.89 \pm 8.01 \mathrm{ug} / \mathrm{dl}$ and the plasma levels of prolactin were $8.28 \pm 1.67$ and $19.53 \pm 3.75 \mathrm{ug} / \mathrm{dl}$, respectively. Therefore, the plasma level of cortisol and prolactin were significantly lower in local anesthesia group than placebo group $(\mathrm{P}=0.001$ for cortisol and $\mathrm{P}=0.013$ for prolactin) (Table 2). Paired t test showed, the plasma levels of cortisol and prolactin were decreased significantly in local anesthesia group ( $\mathrm{P}<0.0001$, for both), however were increased significantly in placebo group $(\mathrm{P}<0.0001$, for both) (Figure 2). The mean duration of surgery was recorded in both group and in local anesthesia and placebo groups were $76.74 \pm 12.76$ and $79.88 \pm 16.48$, respectively, so there was no significant difference between two groups regarding to duration of surgery $(\mathrm{P}=0.47)$. The VAS score was recorded in baseline, $12,24,48$, and $72 \mathrm{hrs}$ after operation, so there was no significant difference between both group in VAS of baseline $(\mathrm{P}=0.74)$, however VAS scores were significantly lower in local anesthesia group than placebo group in $12,24,48$, and $72 \mathrm{hrs}$ after operation ( $\mathrm{P}<0.0001$, for all four). Also we divided patients into two groups with respect requiring to Pethidine (analgesic drug) therefore needed rescues Pethidine in local anesthesia and placebo groups were $25 \%$ and $79.2 \%$, respectively and this correlation was significant $(\mathrm{P}<0.0001)$ (Table 1). In addition, there was 
any adverse effect in local anesthesia group.

Table 1: demographic and operational information of patient in both groups.

\begin{tabular}{|c|c|c|c|c|}
\hline \multicolumn{2}{|c|}{ characters } & $\begin{array}{l}\text { Local anes- } \\
\text { thesia group }\end{array}$ & $\begin{array}{l}\text { Placebo } \\
\text { group }\end{array}$ & p-value \\
\hline \multicolumn{2}{|c|}{$\begin{array}{l}\text { Age }(\text { year }) \\
(\text { mean } \pm \text { SD) }\end{array}$} & $35.92 \pm 9.65$ & $35.46 \pm 9.61$ & $0.87 *$ \\
\hline \multicolumn{2}{|c|}{$\begin{array}{l}\text { BMI }(\text { mean } \pm \text { SD }) \\
\left(\mathrm{Kg} / \mathbf{m}^{2}\right)\end{array}$} & $23.75 \pm 3.82$ & $24.69 \pm 3.17$ & $0.40^{*}$ \\
\hline \multicolumn{2}{|c|}{$\begin{array}{l}\text { Duration of } \\
\text { operation (minutes) } \\
(\text { mean } \pm \text { SD) }\end{array}$} & $76.74 \pm 12.76$ & $79.88 \pm 16.48$ & $0.47 *$ \\
\hline \multirow{5}{*}{$\begin{array}{l}\text { VAS } \\
\text { score }\end{array}$} & Base line & $7.41 \pm 1.31$ & $7.50 \pm 0.88$ & $0.74 * *$ \\
\hline & $12 \mathrm{hrs}$ & $4.70 \pm 1.12$ & $6.37 \pm 0.87$ & $<0.0001 * *$ \\
\hline & 24hrs & $3.04 \pm 0.95$ & $5.45 \pm 0.83$ & $<0.0001 * *$ \\
\hline & $48 \mathrm{hrs}$ & $0.70 \pm 0.55$ & $2.91 \pm 0.77$ & $<0.0001 * *$ \\
\hline & $72 \mathrm{hrs}$ & $0.29 \pm 0.46$ & $0.95 \pm 0.62$ & $<0.0001 * *$ \\
\hline \multirow{2}{*}{$\begin{array}{l}\text { rescues } \\
\text { analgesic }\end{array}$} & Yes & $6(25 \%)$ & $19(79.2 \%)$ & $<0.001 * * *$ \\
\hline & No & $18(75 \%)$ & $5(20.8 \%)$ & \\
\hline
\end{tabular}

*Independent $\mathrm{t}$ test, $* *$ Man-Whitney test, ${ }^{* * *}$ Chi Square test, BMI: body mass index, VAS: Visual Analog Scale
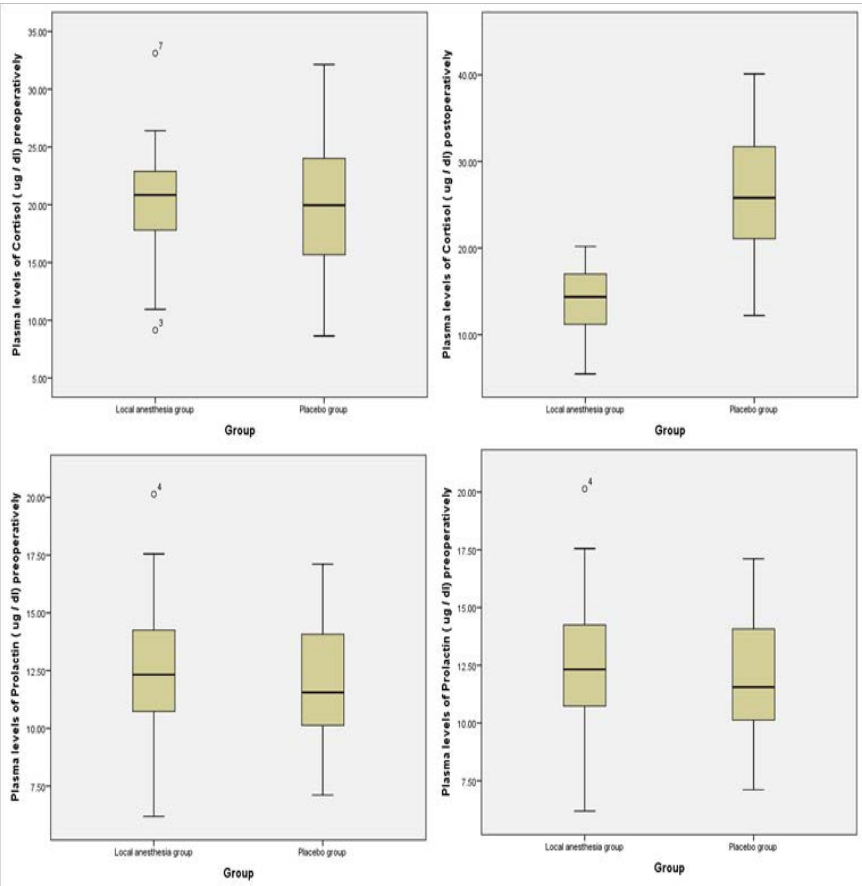

Figure 2: Plasma level of cortisol and prolactin changing in both groups in pre and post operationally.

Table 2: Cortisol and prolactin levels in each group in pre and post operational.

\begin{tabular}{|l|l|l|l|l|}
\hline $\begin{array}{l}\text { Plasma level } \\
\text { (Mean } \pm \text { SD) }\end{array}$ & ( ug / dl) & $\begin{array}{l}\text { Local } \\
\text { anesthesia } \\
\text { group }\end{array}$ & $\begin{array}{l}\text { Placebo } \\
\text { group }\end{array}$ & $\begin{array}{l}\text { p- } \\
\text { value* }\end{array}$ \\
\hline $\begin{array}{l}\text { Pre } \\
\text { operational }\end{array}$ & Cortisol & $20.24 \pm 5.18$ & $19.94 \pm 6.52$ & 0.17 \\
\cline { 2 - 5 } & Prolactin & $12.47 \pm 3.36$ & $11.84 \pm 2.76$ & 0.65 \\
\hline $\begin{array}{l}\text { Post } \\
\text { operational }\end{array}$ & Cortisol & $13.96 \pm 3.64$ & $26.89 \pm 8.01$ & 0.001 \\
\cline { 2 - 5 } & Prolactin & $8.28 \pm 1.67$ & $19.53 \pm 3.75$ & 0.013 \\
\hline
\end{tabular}

\section{Discussion}

Local anesthesia for inguinal hernia is a simple, safe, effective and without complications that suggested to control of postoperative pain ${ }^{[12]}$. In this current study, the mean plasma levels of cortisol and prolactin were decreased in local anesthesia group, also VAS scores and needed rescues analgesic were significantly lower in local anesthesia group. Therefore, local anesthesia may have a suppression effect on stress hormones, postoperative pain and needed rescues analgesic. In the recently studies local anesthesia was recommended for inguinal hernia repair as feasible, effective and safe procedure ${ }^{[13,14]}$. Also local anesthesia had more advantages than general anesthesia in hernia repairing in a prospective study by Friemert ${ }^{[15]}$. In the randomized controlled study by Schmitz ${ }^{[16]}$ who evaluated 45 patients were under general anesthesia along with local anesthesia in tension free hernia repair, indicated that pain decreased until after surgery in administering local anesthesia. Kurmann and collagenous ${ }^{[17]}$ indicated intraoperative infiltration of local anesthesia not had effect on suppression of chronic postoperative pain, the results of this study was in contrast with our study. Furthermore, the study by Solak ${ }^{[18]}$ who evaluated the effects of caudal anesthesia on cortisol and prolactin responses in assessment of postoperative pain in children, so caudal anesthesia had a significant role in attenuating cortisol and prolactin secretion and postoperative pain and no serious adverse effects were reported. In our study bupivacaine was used for local anesthesia. In a study, Teyin and colleagues ${ }^{[19]}$, compared the effects of bupivacaine and morphine on endocrine response in the children undergoing caudal anesthesia along with general anesthesia, therefore they indicated that bupivacaine was more effective than morphine in reducing postoperative stress response. In another study effects of ropivacaine infiltration on postoperative cortisol and prolactin in children undergoing herniorrhaphy were investigated by Sakellaris ${ }^{[20]}$, the results of this study were also in line with our study that cortisol and prolactin and postoperative pain reduced local infiltration. One more study also suggested the used of local wound infiltration is a reliable, safe and satisfaction for the surgeon after herniorrhaphy in according to local wound Infiltration or caudal block ${ }^{[21]}$. In our study patients were divided into two groups with respect requiring to Pethidine drug (up to 72 hours after surgery), the need of analgesic medication was lower in patients undergoing local anesthesia. In the study by Tverskoy et $\mathrm{al}^{[22]}$, they concluded that local anesthetics reduced the need for opioids in the postoperative and even it reduced excessive postoperative pain for 10 days after evaluating the effects of local anesthetics on the reduction of postoperative opioid consumption. In a meta-analysis study that performed by Wu et al. authors suggested local anesthesia for killing pain after hernia repair with low rescue analgesia and without complication. Also in our study local anesthesia infiltration had more effect on postoperative pain $^{[23]}$.

\section{Conclusion}

In conclusion, we showed that local anesthesia in the herniorrhaphy reduced the cortisol and prolactin hormones, postoperative pain and analgesic drugs consumption. We also indicated that our results were in line with previous studies and confirmed local anesthesia as a safe, effective and feasible method and without complication for herniorrhaphy. The limitations of our study were low sample size and not considering other influential factors. Also we need more study to confirm our results. In the final, we recommend local anesthesia as an effective, safe methods and without complications for patients undergoing herniorrhaphy.

Conflict of interest: All authors don't have conflict of interest.

*Independent $t$ test 
Citation: Kolahdouzan, M., et al. Administering of Local Anesthesia with Bupivacaine in the Patients Undergoing Inguinal Hernia Repair. (2018) J Anesth Surg 5(1): $71-74$.

\section{References}

1. McGrath, B., Elgendy, H., Chung, F., et al. Thirty percent of patients have moderate to severe pain $24 \mathrm{hr}$ after ambulatory surgery: a survey of 5,703 patients. (2004) Can J Anaesth 51(9): 886-891. PubMed | CrossRef $\mid$ Others

2. Olsson, A., Sandblom, G., Fränneby, U., et al. Impact of postoperative complications on the risk for chronic groin pain after open inguinal hernia repair. (2017) Surgery 161(2): 509-516.

PubMed | CrossRef | Others

3. Bærentzen, F., Maschmann, C., Jensen, K., et al. Ultrasound-guided nerve block for inguinal hernia repairs: a randomized, controlled, double-blind study. (2012) Reg Anesth Pain Med 37(5): 502-507.

PubMed | CrossRef | Others

4. Waechter, F.L., Sampaio, J.A., Pinto, R.D., et al. A comparison between topical and infiltrative bupivacaine and intravenous meperidine for postoperative analgesia after inguinal herniorrhaphy. (2001) Am Surg 67(5): 447.

PubMed |CrossRef |Others

5. Acevedo, A., Leon, J. Ambulatory hernia surgery under local anesthesia is feasible and safe in obese patients. (2010) Hernia 14(1): 57-62.

PubMed |CrossRef | Others

6. Tanz, J.S., McClintock, M.K. The Physiologic Stress Response during Mediation. (2017) Ohio St J Disp Resol 32: 29.

PubMed |CrossRef $\mid$ Others

7. Fraioli, M.F., Umana, G., Pagano, A., et al. Prolactin Secreting Pituitary Microadenoma: Results of Transsphenoidal Surgery after Medical Therapy With Dopamine Agonist. (2017) J Craniofac Surg 28(4): 992-994.

PubMed | CrossRef|Others

8. Mohapatra, S., Nayak, S.R., Jena, P., et al. A Study of Changes in Stress Factors during Elective Upper Abdominal Surgery Using Different Anaesthetic Techniques. (2016) Int J Pharm Sci Rev Res 39(1): 167-172.

PubMed | CrossRef $\mid$ Others

9. Langouche, L., Van den Berghe, G. Hypothalamic-pituitary hormones during critical illness: A dynamic neuroendocrine response. (2014) Clin Neurol 124: 115-126. PubMed | CrossRef | Others

10. Taylor, L.K., Auchus, R.J., Baskin, L.S., et al. Cortisol response to operative stress with anesthesia in healthy children. (2013) J Clin Endocrinol Metab 98(9): 3687-3693.

PubMed | CrossRef | Others

11. Bringman, S., Holmberg, H., Österberg, J. Location of recurrent groin hernias at TEP after Lichtenstein repair: a study based on the Swedish Hernia Register. (2016) Hernia 20(3): 387-391.

PubMed | CrossRef | Others

12. Amid, P.K., Shulman, A.G., Lichtenstein, I.L. Local anesthesia for inguinal hernia repair step-by-step procedure. (1994) Ann Surg 220(6): 735-737.

PubMed | CrossRef | Others
13. Olsha, O., Feldman, A., Odenheimer, D.B., et al. Local anesthesia for inguinal hernia repair in adolescents. (2007) Hernia 11(6): 497-500.

PubMed | CrossRef $\mid$ Others

14. Ribeiro, F.A.d.S., et al. Inguinal hernia repair with local anesthesia in the outpatient. (2010) Rev Col Bras Cir 37(6): 397-402.

PubMed |CrossRef|Others

15. Friemert, B., Faoual, J., Hölldobler, G., et al. A prospective randomized study on inguinal hernia repair according to the Shouldice technique. Benefits of local anesthesia. (2000) Chirurg 71(1): 52-57.

PubMed $\mid$ CrossRef $\mid$ Others

16. Schmitz, R., Shah, S., Treckmann, J., et al. Extraperitoneal," tension free" inguinal hernia repair with local anesthesia--a contribution to effectiveness and economy. (1997) Langenbecks Arch Chir Suppl Kongressbd 114: 1135-1138.

PubMed | CrossRef $\mid$ Others

17. Kurmann, A., Fischer, H., Dell-Kuster, S., et al. Effect of intraoperative infiltration with local anesthesia on the development of chronic pain after inguinal hernia repair: a randomized, triple-blinded, placebo-controlled trial. (2015) Surgery 157(1): 144154.

PubMed | CrossRef $\mid$ Others

18. Solak, M., Ulusoy, H., Sarihan, H. Effects of caudal block on cortisol and prolactin responses to postoperative pain in children. (2000) Eur J Pediatr Surg 10(4): 219-223.

PubMed $\mid$ CrossRef $\mid$ Others

19. Teyin, E., et al. The efficacy of caudal morphine or bupivacaine combined with general anesthesia on postoperative pain and neuroendocrine stress response in children. (2006) Pediat Anesth 16(3): 290-296.

PubMed |CrossRef | Others

20. Sakellaris, G., Derbent, A., Balcioglu, T., et al. Effects of ropivacaine infiltration on cortisol and prolactin responses to postoperative pain after inguinal hernioraphy in children. (2004) J Pediat Surg 39(9): 1400-1403.

PubMed | CrossRef | Others

21. Gavrilovska-Brzanov, A., Kuzmanovska, B., Kartalov, A., et al. Evaluation of Anesthesia Profile in Pediatric Patients after Inguinal Hernia Repair with Caudal Block or Local Wound Infiltration. (2016) Open Access Maced J Med Sci 4(1): 89-93.

PubMed | CrossRef $\mid$ Others

22. Tverskoy, M., Cozacov, C., Ayache, M., et al. Postoperative pain after inguinal herniorrhaphy with different types of anesthesia. (1990) Anesth Analg 70(1): 29-35.

PubMed | CrossRef $\mid$ Others

23. Wu, C.-C., Bai, C.H., Huang, M.T., et al. Local anesthetic infusion pump for pain management following open inguinal hernia repair: a meta-analysis. (2014) Int J Surg 12(3): 245-250.

PubMed | CrossRef | Others 